\title{
Formalising Behaviour Preserving Program Transformations
}

\author{
Tom Mens ${ }^{1}$, Serge Demeyer ${ }^{2}$, and Dirk Janssens ${ }^{2}$ \\ 1 Postdoctoral Fellow of the Fund for Scientific Research - Flanders \\ Programming Technology Lab, Vrije Universiteit Brussel, \\ Pleinlaan 2, B-1050 Brussel, Belgium \\ tom.mensevub.ac.be \\ 2 Department of Mathematics and Computer Science, Universiteit Antwerpen, \\ Universiteitsplein 1, B-2610 Antwerpen, Belgium \\ \{serge.demeyer, dirk.janssens\}@ua.ac.be
}

\begin{abstract}
The notion of refactoring - transforming the source-code of an objectoriented program without changing its external behaviour- has increased the need for a precise definition of refactorings and their properties. This paper introduces a graph representation of those aspects of the source code that should be preserved by a refactoring, and graph rewriting rules as a formal specification for the refactoring transformations themselves. To this aim, we use type graphs, forbidden subgraphs, embedding mechansims, negative application conditions and controlled graph rewriting. We show that it is feasible to reason about the effect of refactorings on object-oriented programs independently of the programming language being used. This is crucial for the next generation of refactoring tools.
\end{abstract}

\section{Introduction}

Refactorings are software transformations that restructure an object-oriented program while preserving its behaviour [1-3]. The key idea is to redistribute instance variables and methods across the class hierarchy in order to prepare the software for future extensions. If applied well, refactorings improve the design of software, make software easier to understand, help to find bugs, and help to program faster [1].

Although it is possible to refactor manually, tool support is considered crucial. Tools such as the Refactoring Browser support a semi-automatic approach [4], which is recently being adopted by industrial strength software development environments (e.g., VisualWorks, TogetherJ, JBuilder, Eclipse ${ }^{3}$ ). Other researchers demonstrated the feasibility of fully automated tools [5]; studied ways to make refactoring tools less dependent on the implementation language being used [6] and investigated refactoring in the context of a UML case-tool [7].

Despite the existence of such tools, the notion of behaviour preservation is poorly defined. This is mainly because most definitions of the behaviour of an object concentrate on the run-time aspects while refactoring tools must necessarily restrict themselves to the static description as specified in the source-code. Refactoring tools typically rely

\footnotetext{
${ }^{3}$ see http: / / www.refactoring.com/ for an overview of refactoring tools
} 
on an abstract syntax tree representation of the source-code and assert pre- and postconditions before and after transforming the tree [8]. This representation contains details about the control flow of a program, which are largely irrelevant when specifying the effects of a refactoring on the program structure. Moreover, an abstract syntax tree is necessarily dependent on the programming language being used, while refactorings should be defined independently of the programming language [6].

For these reasons, a lightweight graph representation of the source code is probably more appropriate for studying refactorings. Such a representation should not bother with the details necessary for sophisticated data- and control flow analysis or type inferencing techniques, since these are necessarily dependent on the programming language. Instead it should focus on the core concepts present in any class-based object-oriented language -namely classes, methods and variables- and allow us to verify whether the relationships between them are preserved. Moreover, it should enable a transparant yet formal specification of the refactorings, as direct manipulations of the graph representation.

Therefore, this paper presents a feasibility study to see whether graph rewriting can be used to formalise what exactly is preserved when performing a refactoring. Section 2 introduces the concept of refactorings by means of a small motivating example and presents several types of behaviour that should be preserved. In Section 3 we introduce the typed graph representation of the source code and formalise two selected refactorings ("encapsulate field" and "pull up method") by graph rewriting productions. In Section 4 we use this formalisation to guarantee the preservation of well-formedness and certain types of behaviour. Section 5 concludes the paper with the lessons learned regarding the feasibility of graph rewriting as a formal basis for the behaviour preserved during refactoring.

\section{Motivating example}

As a motivating example, this paper uses a simulation of a Local Area Network (LAN). The example has been used successfully by the Programming Technology Lab of the Vrije Universiteit Brussel and the Software Composition Group of the University of Berne to illustrate and teach good object-oriented design. The example is sufficiently simple for illustrative purposes, yet covers most of the interesting constructs of the object-oriented programming paradigm (inheritance, late binding, super calls, method overriding). It has been implemented in Java as well as Smalltalk. Moreover, the example follows an incremental development style and as such includes several typical refactorings. Thus, the example is sufficiently representative to serve as a basis for a feasability study.

\subsection{Local Area Network simulation}

In the initial version there are 4 classes: Packet, Node and two subclasses Workstation and PrintServer. The idea is that all Node objects are linked to each other in a token ring network (via the nextNode variable), and that they can send or accept a Packet object. PrintServer and Workstation refine the behaviour of accept (and perform a super call) 
to achieve specific behaviour for printing the Packet (lines 18-20) and avoiding endless cycling of the Packet (lines 26-28). A Packet object can only originate from a WorkStation object, and sequentially visits every Node object in the network until it reaches its addressee that accepts the Packet, or until it returns to its originator workstation (indicating that the Packet cannot be delivered).

Below is some sample Java code of the initial version where all constructor methods have been omitted due to space considerations. Although the code is in Java, other implementation languages could serve just as well, since we restrict ourselves to core object-oriented concepts only.

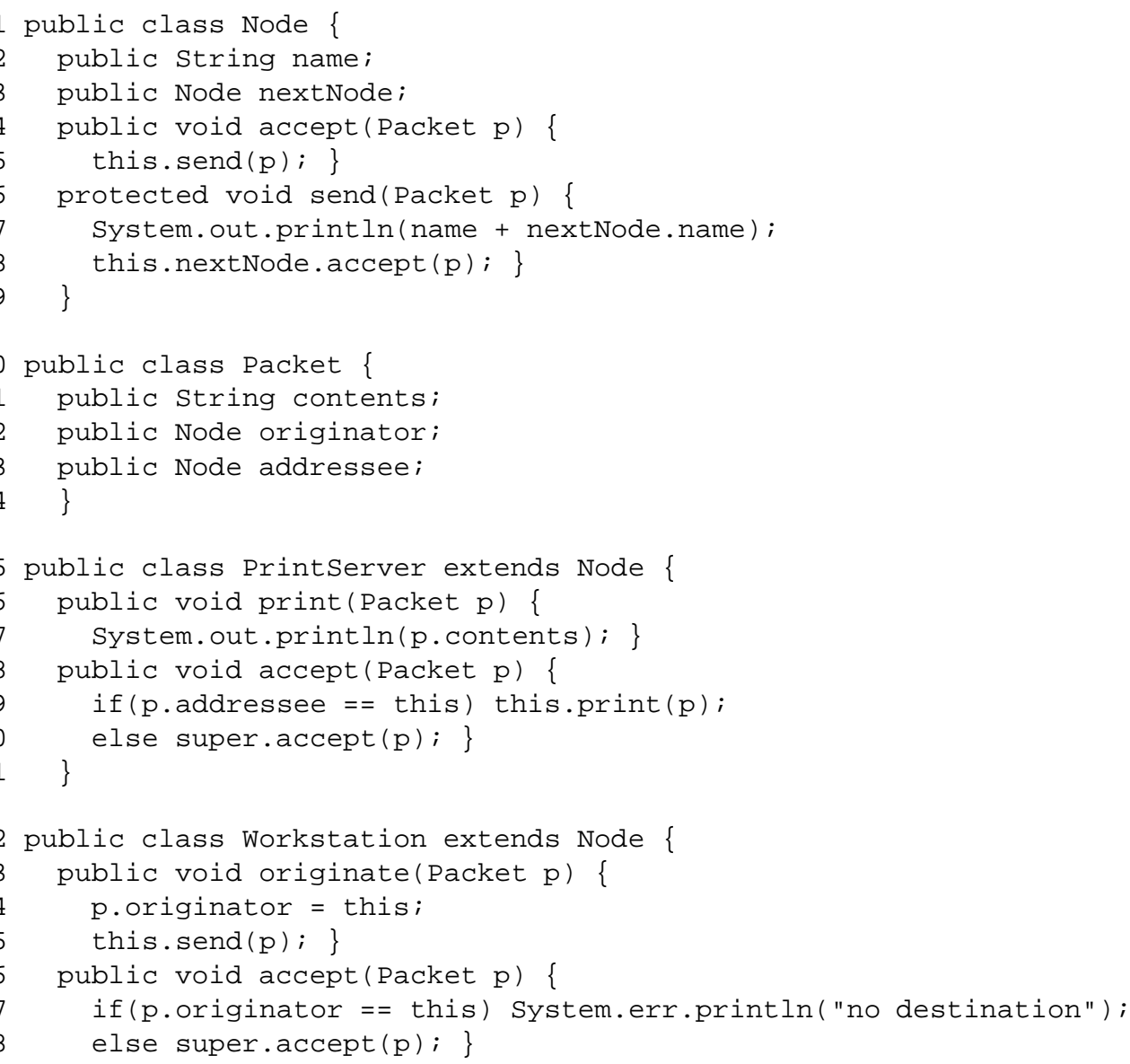

This initial version serves as the basis for a rudimentary LAN simulation. In subsequent versions, new functionality is incorporated incrementally and the object-oriented structure is refactored accordingly. First, logging behaviour is added which results in an "extract method" refactoring ([1], p110) and an "encapsulate field" refactoring ([1], p206). Second, the PrintServer functionality is enhanced to distinguish between ASCII- and PostScript documents, which introduces complex conditionals and requires 
an "extract class" refactoring ([1], p149). The latter is actually a composite refactoring which creates a new intermediate superclass and then performs several "pull up field" $([1], 320)$ and "pull up method" ([1], p322) refactorings. Finally, a broadcast packet is added which again introduces complex conditionals, resolved by means of an "extract class", "extract method", "move method" ([1], p142) and "inline method" ([1], p117).

\subsection{Selected refactorings}

Fowler's catalogue [1] lists seventy-two refactorings and since then many others have been discovered. Since the list of possible refactorings is infinite, it is impossible to prove that all of them preserve behaviour. However, refactoring theory and tools assume that there exist a finite set of primitive refactorings, which can then freely be combined into composite refactorings. For this feasability study, we restrict ourselves to two frequently used primitive refactorings, namely "encapsulate field" and "pull up method". The preconditions for these two object-oriented refactorings are quite typical, hence they may serve as representatives for the complete set of primitive refactorings.

EncapsulateField. Fowler [1] introduces the refactoring EncapsulateField as a way to encapsulate public variables by making them private and providing accessors. In other words, for each public variable a method is introduced for accessing ("getting") and updating ("setting") its value, and all direct references to the variable are replaced by dynamic calls (this messages) to these methods.

Precondition. Before creating the new accessing and updating methods on a class $C$, a refactoring tool should verify that no method with the same signature exists in any of $C$ 's ancestors and descendants, $C$ included. Otherwise, the refactoring may accidentally override (or be overridden by) an existing method, and then it is possible that the behaviour is not preserved.

PullUpMethod. Fowler [1] introduces the refactoring PullUpMethod as a way to move similar methods in subclasses into a common superclass. This refactoring removes code duplication and increases code reuse by inheritance.

Precondition. When a method $m$ with signature $s$ is pulled up into a class $C$, it implies that all methods with signature $s$ defined on the direct descendants of $C$ are removed and replaced by a single occurrence of $m$ now defined on $C$. However, a tool should verify that the method $m$ does not refer to any variables defined in the subclass. Otherwise the pulled-up method would refer to an out-of-scope variable and then the transformed code would not compile. Also, no method with signature $s$ may exist on $C$, because a method that is overwritten accidentally may break the existing behaviour.

\subsection{Behaviour preservation}

Since we take a lightweight approach to source code refactoring here, we only look at notions of behaviour preservation that can be detected statically and do not rely on sophisticated data- and control-flow analysis or type inferencing techniques. The general idea is that, for each considered refactoring, one may catalog the types of behaviour that need to be preserved. For the feasibility study of this paper, we concentrate on three 
types of behaviour preservation that are important and non-trivial for the two selected refactorings. Section 4 discusses to which extent the selected refactorings satisfy these preservation properties:

A refactoring is access preserving if each method implementation accesses at least the same variables after the refactoring as it did before the refactoring. These variable accesses may occur transitively, by first calling a method that (directly or indirectly) accesses the variable. A refactoring is update preserving if each method implementation performs at least the same variable updates after the refactoring as it did before the refactoring. A refactoring is call preserving if each method implementation still performs at least the same method calls after the refactoring as it did before the refactoring.

\section{Formalising refactoring by graph rewriting}

\subsection{Graph notation}

\begin{tabular}{|c|c|c|c|}
\hline \multicolumn{3}{|c|}{\begin{tabular}{l|l} 
node type & description
\end{tabular}} & examples \\
\hline & $C$ & Class & Node, Workstation, PrintServer, Packet \\
\hline & $B$ & method Body & System.out.println (p.contents) \\
\hline & $V$ & Variable & name, nextNode, contents, originator \\
\hline & $S$ & method Signature & accept, send, print \\
\hline & $P$ & formal Parameter of a message & $p$ \\
\hline & $E$ & (sub)Expressior & p. contents \\
\hline & edge type & description & examples \\
\hline & $S \rightarrow B$ & dynamic method lookup & accept (Packet $p$ ) has 3 possible method bodies \\
\hline$i$ : & $C \rightarrow C$ & inheritance & class PrintServer extends Node \\
\hline & $V \rightarrow C$ & variable membership & variable name belongs to Node \\
\hline & $B \rightarrow C$ & method membership & method send is implemented in Node \\
\hline$t$ & $P \rightarrow C$ & message parameter type & print (Packet p) \\
\hline & $V \rightarrow C$ & variable type & String name \\
\hline & $S \rightarrow C$ & signature return type & String get Name() \\
\hline$p:$ & $S \rightarrow P$ & formal parameter & send (Packet $\mathbf{p})$ \\
\hline & $E \rightarrow E$ & actual parameter & System.out.println (nextNode.name) \\
\hline & $B \rightarrow E$ & expression in method body & $\begin{array}{l}\text { if }(p \cdot \operatorname{addressee}==\text { this) this.print }(\mathrm{p}) \text {; } \\
\text { else super.accept }(\mathrm{p}) \text {; }\end{array}$ \\
\hline & $E \rightarrow E$ & cascaded expression & nextNode.accept (p) \\
\hline$d$ & $E \rightarrow S$ & dynamic method call & this.send $(p)$ \\
\hline$a$ & $E \rightarrow P$ & parameter access & p.originator \\
\hline & $E \rightarrow V$ & variable access & p.originator \\
\hline$u:$ & $E \rightarrow V$ & variable update & p.originator $=$ this \\
\hline
\end{tabular}

Table 1. Node type set $\Sigma=\{C, B, V, S, P, E\}$ and edge type set $\Delta=\{l, i, m, t, p, e, \bullet, d, a, u\}$ 
The graph representation of the source code is rather straightforward. Software entities (such as classes, variables, methods and method parameters) are represented by nodes whose label is a pair consisting of a name and a node type. For example, the class Packet is represented by a node with name Packet and type $C$ (i.e., a $C$-node). The set $\Sigma=\{C, B, V, S, P, E\}$ of all possible node types is clarified in Table 1. Method bodies ( $B$-nodes) have been separated from their signatures ( $S$-nodes) to make it easier to model late binding and dynamic method lookup, where the same signature may have many possible implementations. $B$-nodes (method bodies) and $P$-nodes (formal parameters) have an empty name.

Relationships between software entities (such as membership, inheritance, method lookup, variable accesses and method calls) are represented by edges between the corresponding nodes. The label of an edge is simply the edge type. For example, the inheritance relationship between the classes Workstation and Node is represented by an edge with type $i$ (i.e., an $i$-edge) between the $C$-nodes Workstation and Node. The set $\Delta=\{l, i, m, t, p, e, \boldsymbol{\bullet}, d, a, u\}$ of all possible edge types is clarified in Table 1. For $m$-edges (membership), the label is often omitted in the figures.

Using this notation, an entire program can be represented by means of a single typed graph. Because the graph representation can become very large, we only display those parts of the graph that are relevant for the discussion. For example, Figure 1 only shows the graph representation of the static structure of the LAN simulation. (For $B$-nodes, a name has been put between parentheses to make the graph more readable.)

A method body is represented by a structure consisting of $E$-nodes connected by edges that express information about dynamic method calls and variable invocations (accesses and updates). For example, Figure 2 represents the method bodies in class Node. The method body of send contains a sequence of two subexpressions, which is denoted by two $e$-edges from the $B$-node to two different $E$-nodes. The second subexpression nextNode. accept $(p)$ is a cascaded method call (represented by a $\bullet$-edge) consisting of a variable access (represented by an $a$-edge to the $V$-node labelled next $N$ ode) followed by a dynamic method call with one parameter (represented by a $d$-edge and corresponding $p$-edge originating from the same $E$-node).

We have deliberately kept the graph model very simple to make it as language independent as possible. It does not model Java-specific implementation features such as: Java interfaces; explicit references to this; constructor methods; control statements (such as if, for, etc.); Java modifiers (such as static, abstract, protected, final); inner classes; threads; exceptions.

\subsection{Well-formedness constraints}

On top of the graph representation, we need to impose constraints to guarantee that a graph is well-formed in the sense that it corresponds to a syntactically correct program. These well-formedness constraints are essential to fine-tune our graph notation to a particular programming language (in this case Java). We use two mechanisms to express these constraints: a type graph and forbidden subgraphs.

The notion of a type graph (or graph schema) is formally presented in $[9,10]$. Intuitively, a type graph is a meta-graph expressing restrictions on the instance graphs that are allowed. Formally, a graph is well-formed only if there exists a graph morphism into 


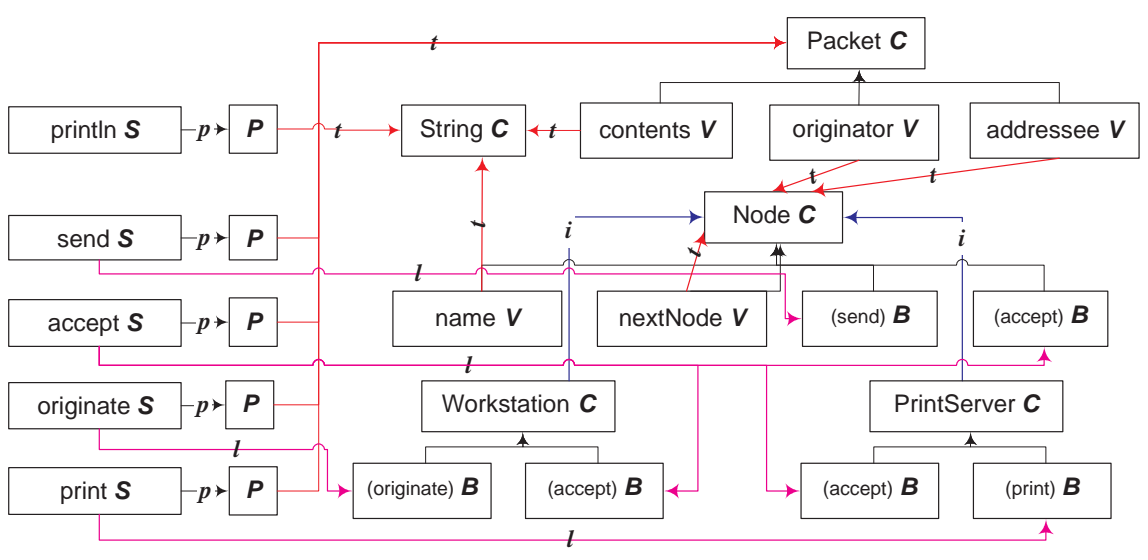

Fig. 1. Static structure of LAN simulation

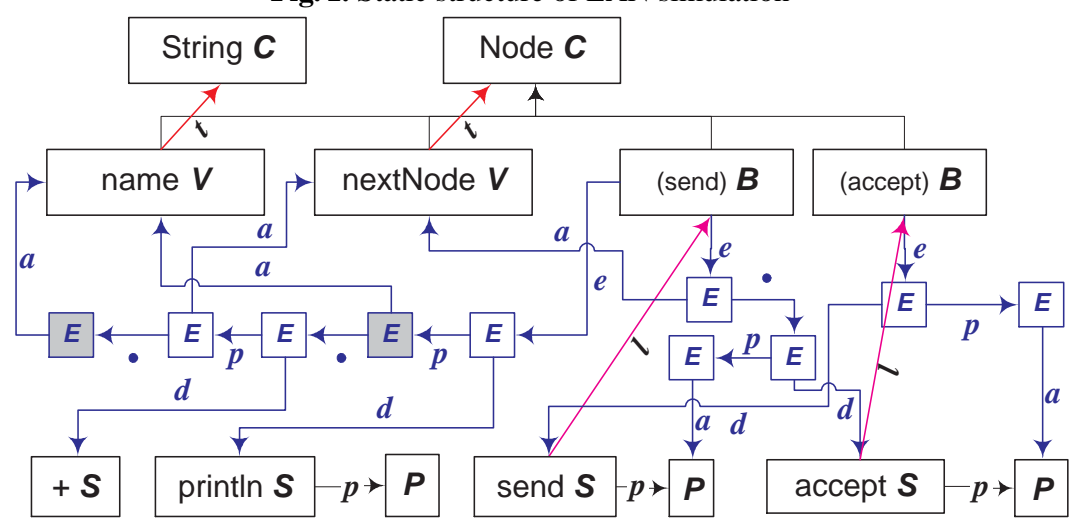

Fig. 2. Method bodies of class Node

the type graph: a node mapping and edge mapping that preserves sources, targets and labels. For node labels, only the type component, as introduced in Table 1, is taken into account. Figure 3 displays the type graph needed for our particular graph representation.

Because type graphs alone are insufficient to express all constraints that we are interested in, we use a second mechanism called forbidden subgraphs to exclude illegal configurations in a graph. A graph $G$ satisfies the constraint expressed by a forbidden subgraph $F$ if there does not exist an injective graph morphism from $F$ into $G$. To specify forbidden subgraphs we use path expressions of the form $a \underset{e x p}{\longrightarrow} b$, where $a$ and $b$ belong to the node type set $\Sigma$ of Table 1, and $\exp$ is a regular expression over the edge type set $\Delta$ of Table $1 .{ }^{4}$ A graph that contains more than one path expression denotes the set of all graphs obtained by substituting these edges in the obvious way.

${ }^{4}$ This is a simplified use of the path expressions provided by PROGRES [11]. 


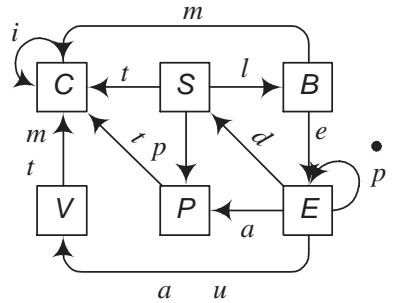

Fig. 3. Type Graph

Some typical examples of well-formedness constraints, needed to guarantee that a refactoring does not lead to an ill-formed graph, are given below:

WF-1 A variable cannot be defined in a class if there is already a variable with the same name in the inheritance hierarchy of this class (i.e., in the class itself or in an ancestor class or descendant class).

WF-2 A method with the same signature cannot be implemented twice in the same class.

WF-3 A method in a class cannot refer to variables that are defined in its descendant classes.

These constraints can be expressed by the forbidden subgraphs of Figure $4 .^{5}$ The forbidden subgraph for WF-3 uses two path expressions. Expression $B \underset{\text { ? }^{*}\{a \mid u\}}{\longrightarrow} V$ denotes the set of all nonempty edge paths from a $B$-node to a $V$-node, where the last edge must have either type $a$ or type $u$. It specifies the existence of an update or access of a variable from within the given method body.

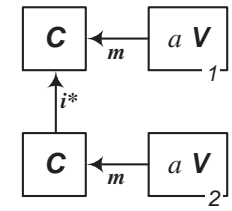

$W F-1$

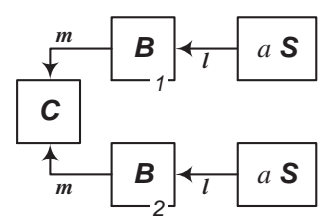

WF-2

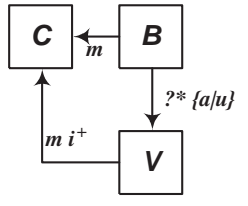

$W F-3$

Fig. 4. Well-formedness constraints expressed as forbidden subgraphs

\footnotetext{
${ }^{5}$ Nodes identified by a different number in the forbidden subgraph are mapped onto different nodes in the graph.
} 


\subsection{Graph rewriting productions}

A graph rewriting is a transformation that takes an initial graph as input and transforms it into a result graph. This transformation occurs according to some predefined rules that are specified in a so-called graph production. Such a graph production is specified by means of a left-hand side (LHS) and a right-hand side (RHS). The LHS is used to specify which parts of the initial graph should be transformed, while the RHS specifies the result after the transformation. Often, a graph production can be applied to different parts of a graph, leading to different occurrences (or matches) of the graph production's LHS. In this paper, we use parameterised graph productions that contain variables for labels. Such parameterised productions can be instantiated by assigning concrete values to the variables.

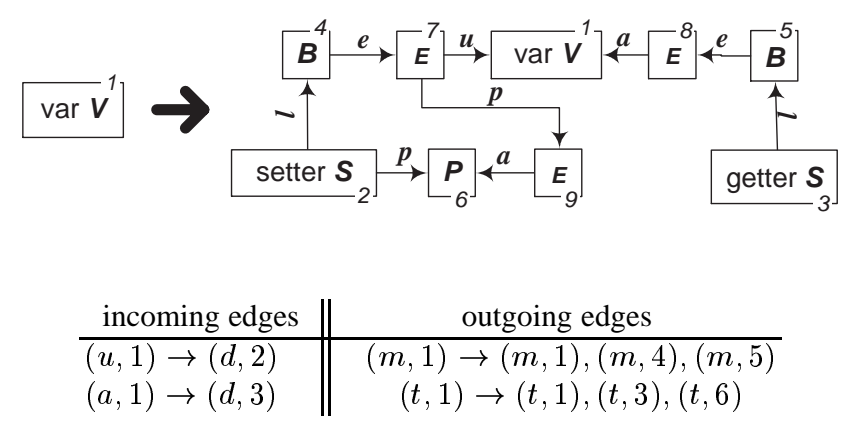

Fig. 5. Parameterised graph production EncapsulateField(var,accessor,updater) with embedding table

Figure 5 shows a production where var, accessor and updater are such variables. This production represents the refactoring EncapsulateField. LHS and RHS are separated by means of an arrow symbol. All nodes are numbered. Nodes that have a number occurring in both the LHS and the RHS are preserved by the rewriting (e.g., node 1). Nodes with numbers that only occur in the LHS are removed, and nodes with numbers that only occur in the RHS (e.g., nodes 2 and 3) are newly created.

In order to take into account the "context" in which the production is applied, consisting of the (sub)expressions referring to the variable that is encapsulated, the production is equipped with an embedding mechanism similar to the one of [12]. ${ }^{6}$ This embedding mechanism specifies how edges are redirected. Incoming edges, i.e., edges that have their target node in the LHS but not their source node, are redirected according to the incoming edges specification in Figure 5. For example, $(u, 1) \rightarrow(d, 2)$ means that each update of the variable var (represented by an incoming $u$-edge to node 1 ) is replaced by a dynamic method call to the updater method (represented by an incoming $d$-edge to node 2). Outgoing edges are treated similarly, using the outgoing edges specification in Figure 5. For example, $(m, 1) \rightarrow(m, 1),(m, 4),(m, 5)$ means that the

\footnotetext{
${ }^{6}$ A similar but more visual mechanism is available in PROGRES [11] and Fujaba [13].
} 
method bodies (nodes 4 and 5) that correspond to the accessor and updater signature must be implemented in the same class as the one in which the variable var (node 1) was defined.

The parameterised production of Figure 5 may be viewed as a specification of an infinite set of productions in the algebraic approach to graph rewriting [14, 15]. A concrete production can be obtained by filling in the variables of the parameterised graph production with concrete values and extending the LHS and RHS of the embedding-based production with a concrete context. Figure 6 shows the production instance EncapsulateField(name,getName,setName) that is applied in the context of the LAN example of Figure 2. The two gray $E$-nodes in Figure 6 are matched with the gray $E$-nodes of Figure 2.

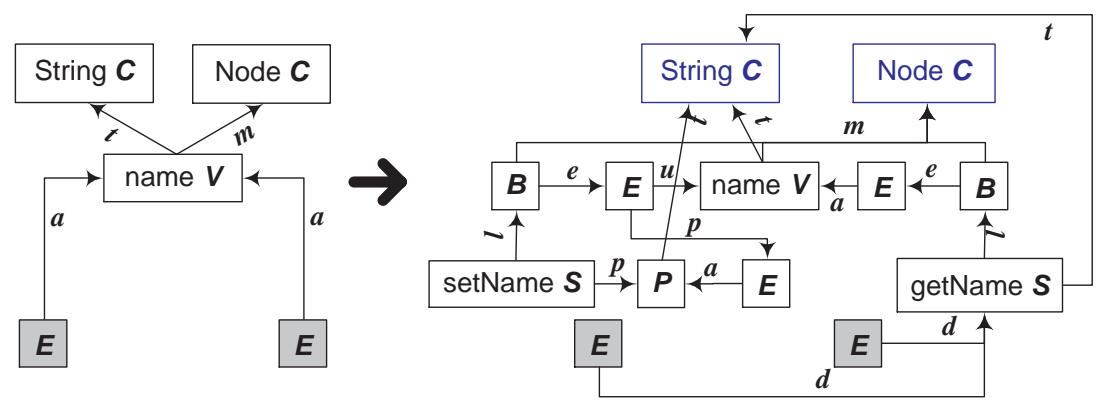

Fig. 6. Graph production instance EncapsulateField(name,getName, setName) obtained from the parameterised production of Figure 5

The second refactoring that we want to express is PullUpMethod(parent,child,name), which moves the implementation of a method name in some child class to its parent class, and removes the implementations of the method name in all other children of parent. Expressing this refactoring by a single production -even a parameterised one with embedding mechanism-is problematic: changes may have to be made in all subclasses of parent, and the number of such subclasses is not a priori bounded. A way to cope with the problem is to control the order in which productions are applied. Mechanisms for controlled graph rewriting (also known as programmed or regulated graph rewriting) have been studied in, e.g., [16-18]. Using these mechanisms, PullUpMethod can be expressed by two parameterised productions $P_{1}$ and $P_{2} . P_{1}$ moves the method name one level higher in the inheritance hierarchy (i.e., from child to parent), and can only be applied if it is immediately followed by an application of $P_{2}$. This second production removes the implementation of method name from another subclass of parent, and has to be applied until there are no more occurrences of its LHS present. 
Both productions $P_{1}$ and $P_{2}$ are equipped with an identity embedding, i.e., all incoming and outgoing edges are preserved.

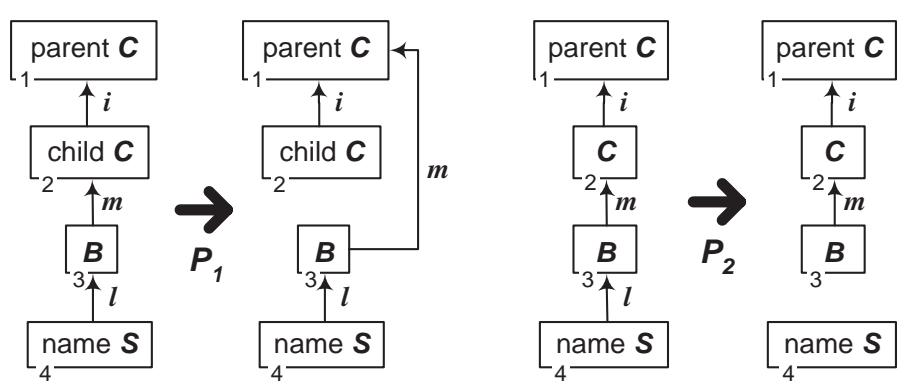

Fig. 7. Productions $P_{1}$ and $P_{2}$ for controlled rewriting PullUpMethod(parent,child,name)

\section{Preservation of refactoring properties}

In this section we combine the formalisation of refactorings of Subsection 3.3 with another graph rewriting technique, negative application conditions, to guarantee certain properties of the graphs that are derived. In particular, we consider certain types of behaviour preservation, refactoring preconditions and well-formedness constraints.

\subsection{Preserving behaviour}

The types of behaviour preservation discussed in Subsection 2.3 can be expressed formally using the path expression notation of Subsection 3.2.

The path expression $B \underset{?^{* *} a}{\longrightarrow} V$ can be used to express the property of access preservation. It specifies all possible access paths from a method body ( $B$-node) to a variable ( $V$-node). The constraints imposed by the type graph of Figure 3 guarantee that there is only a single $a$-edge on such a path, and this edge is the last one in the path. Access preservation means that, for each occurrence of $B \underset{?^{*} a}{\longrightarrow} V$ in the initial graph to be rewritten, there is a corresponding occurrence of this path expression in the resulting graph, connecting the same $B$-node and $V$-node. Thus, the nodes that match $B$ and $V$ should not be removed or added by the graph production. In a similar way, we can express update preservation by means of the expression $B \underset{?^{*} u}{\longrightarrow} V$.

Path expression $B \underset{? * d}{\longrightarrow} S \rightarrow \vec{l} B$ formalises the property of call preservation. For each method body ( $B$-node) that performs a dynamic call ( $d$-edge) to some signature ( $S$-node) in the initial graph, there should still be a method lookup of the same method body in the resulting graph. In general, this requirement is not sufficient, since we also need to ensure that this method body has not been overridden by another one via late 
binding. Unfortunately, our current notation of path expressions cannot specify this constraint.

EncapsulateField. To show update preservation for EncapsulateField, it suffices to show that the preservation property expressed by $B \underset{?^{*} u}{\longrightarrow} V$ is satisfied for each method body $B$ that updates the variable var that is being encapsulated. It follows from the form of the graph production of Figure 5 that this is the case. This is illustrated in Figure 8, that shows how a direct update of var is replaced by a slightly longer path that still satisfies the property $B \overrightarrow{?^{*} u}$ V. Access preservation can be shown in a similar way. Call preservation is trivial since the refactoring does not change any dynamic method calls or method bodies. (It does add new method signatures and method bodies, but this does not affect existing method calls.)

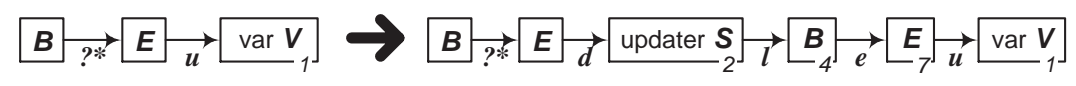

Fig. 8. Update preservation property of EncapsulateField(var,accessor,updater)

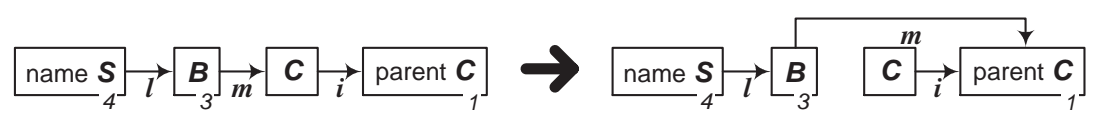

Fig. 9. Call preservation property of PullUpMethod(parent,child,name)

PullUpMethod. To show call preservation for PullUpMethod, we have to check whether the property $B \underset{?^{* *} d}{\longrightarrow} S \underset{l}{\longrightarrow} B$ is preserved by the refactoring. Subexpression $B \underset{?^{*} d}{\longrightarrow} S$ is trivially fulfilled. Subexpression $S \rightarrow \vec{l} B$ is illustrated in Figure 9: all implementations of the signature name by some method body are preserved, even if the class in which this body resides changes to parent. Access preservation and update preservation are trivial except for the case where an implementation of the signature name in some child class of parent accesses or updates a variable. Since this implementation is removed (pulled up) by the refactoring, it is possible that variable accesses or updates in this method implementation are not preserved. Hence, the PullUpMethod refactoring is not necessarily access preserving or update preserving! This can be solved by adding extra preconditions for the refactoring, as shown in the next subsection.

\subsection{Preserving constraints}

In general, the graph obtained by the application of a refactoring production has to satisfy several constraints. On the one hand, it has to satisfy well-formedness constraints, and on the other hand, refactorings are often subject to more specific constraints. For example, EncapsulateField does not cause accidental method overriding, i.e., 
the refactoring may not introduce new methods in a class if these methods are already defined in one of its descendants or ancestors (RC-1)

All these constraints can be expressed in a natural way as postconditions for the graph production. However, for efficiency reasons, it is desirable to transform these postconditions into preconditions. This avoids having to undo the refactoring if it turns out that the constraints are not met. Formally, preconditions can be defined by using graph rewriting with negative application conditions $[19,20]$. Formal proofs are available that specify how postconditions can be transformed into equivalent preconditions for the graph production [20]

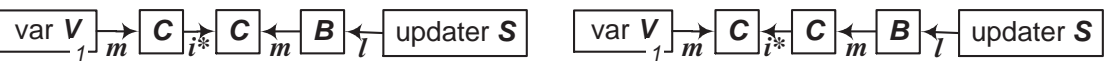

Fig. 10. Negative preconditions for the EncapsulateField refactoring

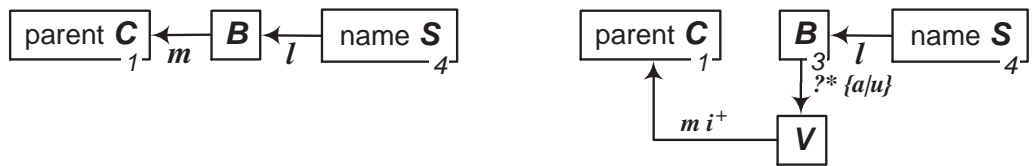

Fig. 11. Negative preconditions for the PullUpMethod refactoring

EncapsulateField. Figure 10 presents the negative preconditions needed in order for EncapsulateField to satisfy refactoring constraint RC-1. The conditions specify that no ancestor or descendant of the class containing var should have implemented a method with signature updater. Two similar negative application conditions are needed for the accessor method. Well-formedness constraint WF-1 is satisfied since EncapsulateField does not introduce or move any variables, or change anything to the class hierarchy. Constraint WF-2 is satisfied thanks to the preconditions of Figure 10, in the special case where $i^{*}$ is the empty word. Constraint WF-3 is satisfied because EncapsulateField only introduces a new variable access and update to a variable that is defined by the class itself.

PullUpMethod. Figure 11 presents two negative preconditions for PullUpMethod, or more specifically, for subproduction $P_{1}$ of Figure 7. The condition on the left specifies that the method name to be pulled up should not yet be implemented in parent, and the condition on the right specifies that the implementation of the method to be pulled up should not refer to (i.e., access or update) variables outside the scope of the parent. PullUpMethod satisfies well-formedness constraint WF-1 since it does not introduce or redirect any variables, or change anything to the class hierarchy. Constraint WF-2 is satisfied thanks to the precondition on the left of Figure 11. Constraint WF-3 is satisfied thanks to the precondition on the right of Figure 11. 


\section{Conclusion and Future Work}

This paper presented a feasibility study concerning the use of graph rewriting as a formal specification for refactoring. Based on the specification of two refactorings ("encapsulate field" and "pull up method") we conclude that graph rewriting is a suitable formalism for specifying the effect of refactorings, because (i) graphs can be used as a language-independent representation of the source code; (ii) rewriting rules are a concise and precise way to specify the source-code transformations implied by a refactoring; (iii) the formalism allows us to prove that refactorings indeed preserve the behaviour that can be inferred directly from the source code.

In order to achieve our goal, we had to combine a number of existing graph rewriting mechanisms and techniques. Type graphs and forbidden subgraphs made it possible to express well-formedness constraints in a natural way. The specification of infinite sets of productions was facilitated by using parameterisation and an embedding mechanism. The application of graph productions was restricted by using negative application conditions and controlled graph rewriting. All these techniques are provided by stateof-the-art graph rewriting tools such as PROGRES [11] and Fujaba [13].

The two refactorings as well as the types of behaviour preservation we studied, are realistic and well documented. Because there are many other types of refactorings and behaviour preservation, further research is needed in order to find out whether the used graph representation needs to be modified, or whether other graph rewriting techniques should be used. Initial attempts to specify refactorings such as "move method" and "push down method" showed that it is difficult to manipulate nested structures in method bodies. Therefore, we need to resort to techniques (such as hierarchical graphs [10] and garbage collection) that tackle the inevitable complexity of large graphs.

A central topic in future work will be the investigation of methods to detect, for a given graph property and graph transformation, whether or not the property is preserved by the transformation. This requires further research into formalisms to express such properties, and to use these in an automated refactoring tool.

Similar to what has been described in [6], we will also study the impact of language specific features (e.g., Java interfaces and exceptions) to verify whether it is possible to express refactorings independently of the programming language being used.

Because tool support is essential to cope with the complexity of refactoring productions, and to automate the checking of behaviour preservation, we are currently implementing our work in a graph rewriting tool. We have also implemented a translator to convert Java source code into our underlying graph representation.

In the longer run, we want to investigate combinations of refactorings. Roberts [8] has argued that primitive refactorings can be chained in sequences, where the preconditions of one refactoring are guaranteed by the postconditions of the previous ones. Moreover, in some refactoring sequences it is possible to change the order without changing the global effect. Such properties can be expressed using graph rewriting formalisms like the one in [20]. Refactoring tools may exploit these properties to optimise the number of program transformations, in much the same way as database tools perform query optimisations. This reduces the amount of analysis that must be performed by a tool, which is crucial for the performance and usability of refactoring tools. 


\section{References}

1. Fowler, M.: Refactoring: Improving the Design of Existing Programs. Addison-Wesley (1999)

2. Opdyke, W.: Refactoring Object-Oriented Frameworks. PhD thesis, University of Illinois at Urbana-Champaign (1992)

3. Opdyke, W., Johnson, R.: Creating abstract superclasses by refactoring. In: Proc. ACM Computer Science Conference, ACM Press (1993) 66-73

4. Roberts, D., Brant, J., Johnson, R.: A refactoring tool for Smalltalk. Theory and Practice of Object Systems 3 (1997) 253-263

5. Casais, E.: Automatic reorganization of object-oriented hierarchies: a case study. Object Oriented Systems 1 (1994) 95-115

6. Tichelaar, S.: Modeling Object-Oriented Software for Reverse Engineering and Refactoring. $\mathrm{PhD}$ thesis, University of Bern (2001)

7. Sunyé, G., Pollet, D., LeTraon, Y., Jézéquel, J.M.: Refactoring UML models. In: Proc. UML 2001. Volume 2185 of Lecture Notes in Computer Science., Springer-Verlag (2001) 134-138

8. Roberts, D.: Practical Analysis for Refactoring. PhD thesis, University of Illinois at UrbanaChampaign (1999)

9. Corradini, A., Ehrig, H., Löwe, M., Montanari, U., Padberg, J.: The category of typed graph grammars and their adjunction with categories of derivations. In: Proceedings 5th International Workshop on Graph Grammars and their Application to Computer Science. Volume 1073 of Lecture Notes in Computer Science., Springer-Verlag (1996) 56-74

10. Engels, G., Schürr, A.: Encapsulated hierarchical graphs, graph types and meta types. Electronic Notes in Theoretical Computer Science 2 (1995)

11. Schürr, A., Winter, A.J., Zündorf, A.: Graph grammar engineering with PROGRES. In Schäfer, W., Botella, P., eds.: Proc. European Conf. Software Engineering. Volume 989 of Lecture Notes in Computer Science., Springer-Verlag (1995) 219-234

12. Janssens, D., Mens, T.: Abstract semantics for ESM systems. Fundamenta Informaticae 26 (1996) 315-339

13. Niere, J., Zündorf, A.: Using Fujaba for the development of production control systems. In Nagl, M., Schürr, A., Münch, M., eds.: Proc. Int. Workshop Agtive 99. Volume 1779 of Lecture Notes in Computer Science., Springer-Verlag (2000) 181-191

14. Ehrig, H.: Introduction to the algebraic theory of graph grammars. In Claus, V., Ehrig, H., Rozenberg, G., eds.: Graph Grammars and Their Application to Computer Science and Biology. Volume 73 of Lecture Notes in Computer Science., Springer-Verlag (1979) 1-69

15. Löwe, M.: Algebraic approach to single-pushout graph transformation. Theoretical Computer Science 109 (1993) 181-224

16. Bunke, H.: Programmed graph grammars. In Claus, V., Ehrig, H., Rozenberg, G., eds.: Graph Grammars and Their Application to Computer Science and Biology. Volume 73 of Lecture Notes in Computer Science., Springer-Verlag (1979) 155-166

17. Kreowski, H.J., Kuske, S.: Graph transformation units and modules. Handbook of Graph Grammars and Computing by Graph Transformation 2 (1999) 607-638

18. Schürr, A.: Logic based programmed structure rewriting systems. Fundamenta Informaticae 26 (1996) 363-385

19. Habel, A., Heckel, R., Taentzer, G.: Graph grammars with negative application conditions. Fundamenta Informaticae 26 (1996) 287-313

20. Heckel, R.: Algebraic graph transformations with application conditions. Master's thesis, TU Berlin (1995) 